\title{
SHARP LORENTZ ESTIMATES FOR DYADIC-LIKE MAXIMAL OPERATORS AND RELATED BELLMAN FUNCTIONS
}

\author{
ANTONIOS D. MELAS AND ELEFTHERIOS N. NIKOLIDAKIS
}

\begin{abstract}
We precisely evaluate Bellman type functions for the dyadic maximal opeator $\mathbb{R}^{n}$ and of maximal operators on martingales related to local Lorentz type estimates. Using a type of symmetrization principle, introduced for the dyadic maximal operator in earlier works of the authors we precisely evaluate the supremum of the Lorentz quasinorm of the maximal operator on a function $\phi$ when the integral of $\phi$ is fixed and also the same Lorentz quasinorm of $\phi$ is fixed. Also we find the corresponding supremum when the integral of $\phi$ is fixed and several weak type conditions are given.
\end{abstract}

Acknowledgement 1. This research has been co-financed by the European Union and Greek national funds through the Operational Program "Education and Lifelong Learning" of the National Strategic Reference Framework (NSRF). ARISTEIA I, MAXBELLMAN 2760, research number 70/3/11913.

Acknowledgement 2. The authors would like to thank Prof. D. Cheliotis for his help.

\section{INTRODUCTION}

The dyadic maximal operator on $\mathbb{R}^{n}$ is defined by

$$
M_{d} \phi(x)=\sup \left\{\frac{1}{|Q|} \int_{Q}|\phi(u)| d u: x \in Q, Q \subseteq \mathbb{R}^{n} \text { is a dyadic cube }\right\}
$$

for every $\phi \in L_{\text {loc }}^{1}\left(\mathbb{R}^{n}\right)$ where the dyadic cubes are the cubes formed by the grids $2^{-N} \mathbb{Z}^{n}$ for $N=0,1,2, \ldots$

As it is well known it satisfies the following weak type $(1,1)$ inequality

$$
\left|\left\{x \in \mathbb{R}^{n}: M_{d} \phi(x)>\lambda\right\}\right| \leq \frac{1}{\lambda} \int_{\left\{M_{d} \phi>\lambda\right\}}|\phi(u)| d u .
$$

for every $\phi \in L^{1}\left(\mathbb{R}^{n}\right)$ and every $\lambda>0$ from which it is easy to get the following $L^{p}$ inequality

$$
\left\|M_{d} \phi\right\|_{p} \leq \frac{p}{p-1}\|\phi\|_{p}
$$

for every $p>1$ and every $\phi \in L^{p}\left(\mathbb{R}^{n}\right)$ which is best possible (see [1, 2] for the general martingales and 20] for dyadic ones).

An approach for studying such maximal operators is the introduction of the so called Bellman functions (see [8]) related to them which reflect certain deeper

Date: March 25, 2014.

2010 Mathematics Subject Classification. [2010] 42B25.

Key words and phrases. Bellman, dyadic, maximal, Lorentz. 
properties of them by localizing. Such functions related to the $L^{p}$ inequality (1.3) have been precisely evaluated in [4. Actually defining for any $p>1$

$\mathcal{B}_{p}(F, f, L)=\sup \left\{\frac{1}{|Q|} \int_{Q}\left(M_{d} \phi\right)^{p}: \operatorname{Av}_{Q}\left(\phi^{p}\right)=F, \operatorname{Av}_{Q}(\phi)=f, \sup _{R: Q \subseteq R} \operatorname{Av}_{R}(\phi)=L\right\}$

where $Q$ is a fixed dyadic cube, $R$ runs over all dyadic cubes containing $Q, \phi$ is nonnegative in $L^{p}(Q)$ and the variables $F, f, L$ satisfy $0 \leq f \leq L, f^{p} \leq F$ which is independent of the choice of $Q$ (so we may take $Q=[0,1]^{n}$ ) it has been shown in 4 that

$$
\mathcal{B}_{p}(F, f, L)=\left\{\begin{array}{cc}
F \omega_{p}\left(\frac{p L^{p-1} f-(p-1) L^{p}}{F}\right)^{p} & \text { if } L<\frac{p}{p-1} f \\
L^{p}+\left(\frac{p}{p-1}\right)^{p}\left(F-f^{p}\right) & \text { if } L \geq \frac{p}{p-1} f .
\end{array}\right.
$$

where $\omega_{p}:[0,1] \rightarrow\left[1, \frac{p}{p-1}\right]$ is the inverse function of $H_{p}(z)=-(p-1) z^{p}+p z^{p-1}$. Actually (see [4) the more general approach of defining Bellman functions with respect to the maximal operator on a nonatomic probability space $(X, \mu)$ equipped with a tree $\mathcal{T}$ (see Section 2) can be taken and the corresponding Bellman function is always the same.

There are several other problems in Harmonic Analysis where Bellman functions naturally arise. Such problems (including the dyadic Carleson imbedding and weighted inequalities) are described in [10] (see also [8, 9] ) and also connections to Stochastic Optimal Control are provided, from which it follows that the corresponding Bellman functions satisfy certain nonlinear second order PDE.

The exact computation of a Bellman function is a difficult task which is connected with the deeper structure of the corresponding Harmonic Analysis problem. Thus far several Bellman functions have been computed (see [1, [2], [4, [12, 13, [16, [17, 18]). L.Slavin and A.Stokolos [15] linked the Bellman function computation to solving certain PDE's of the Monge Ampere type, and in this way they obtained an alternative proof of the Bellman functions relate to the dyadic maximal operator in [4. Also in [18, using the Monge-Ampere equation approach a more general Bellman function than the one related to the dyadic Carleson imbedding Theorem has be precisely evaluated thus generalizing the corresponding result in [4].

However many Bellman functions related to dyadic maximal operators do not obey the dynamics that make the Monge Ampere approach, or the linearization approach readily applicable. Such are the cases related to weak $L^{p}$ as well as more general Lorentz $L^{p, q}$ norms. Recently another approach based on symmetrization, i.e. decreasing rearrangements, was introduced in [5] and then refined in [11] giving results as the computation of the Bellman functions related to mixed local $L^{p} \rightarrow L^{q}$ estimates (see [5]) the determination of sharp constants in $L^{p, \infty} \rightarrow L^{p, \infty}$ and in more general Lorentz $L^{p, q} \rightarrow L^{p, q}$ norm estimates for dyadic maximal operators (see [11) and also another proof of the result in [4] (see [7]). This method is based on the following Theorem essentially proved in [11] (see also [5] for a weaker version) and it refers to the maximal operator $M_{\mathcal{T}}$ defined for any nonatomic probability space $(X, \mu)$, equipped with any tree-like family $\mathcal{T}$ with (see [4]):

Theorem 1. Let $G:[0,+\infty) \rightarrow[0,+\infty)$ be non-decrasing, $h:(0,1] \rightarrow \mathbb{R}^{+}$be any locally integrable function. Then for any nonatomic probability space $(X, \mu)$, 
equipped with any tree-like family $\mathcal{T}$, for any non-increasing right continuous integrable function $g:(0,1] \rightarrow \mathbb{R}^{+}$and any $k \in(0,1]$, the following equality holds:

$$
\begin{gathered}
\sup \left\{\int_{0}^{k} G\left[\left(M_{\mathcal{T}} \phi\right)^{*}(t)\right] h(t) d t: \phi \text { measurable on } X \text { with } \phi^{*}=g\right\}= \\
=\int_{0}^{k} G\left(\frac{1}{t} \int_{0}^{t} g(u) d u\right) h(t) d t .
\end{gathered}
$$

Here $\phi^{*}$ denotes the equimeasurable decreasing rearrangement of the measurable function $\phi: X \rightarrow \mathbb{R}$ which is defined on $(0,1]$ since $X$ is a probability space. For completeness we will give here a simpler proof of the above Theorem. This enables as to reduce the problem of determining a Bellman type function for the local tree maximal operator $M_{\mathcal{T}}$ to a problem of a similar nature but on $(0,1]$ and for the local Hardy operator $\mathcal{H}(g)(t)=\frac{1}{t} \int_{0}^{t} g$ acting on decreasing functions $g$. This idea applied to convex $G$ 's has lead to the determination of the Bellman functions

$$
\begin{gathered}
\mathcal{B}_{p, q}(F, f, L)=\sup \left\{\frac{1}{|Q|} \int_{E}\left(M_{d} \phi\right)^{p}: \operatorname{Av}_{Q}\left(\phi^{p}\right)=F, \operatorname{Av}_{Q}(\phi)=f,\right. \\
\left.\sup _{R: Q \subseteq R} \operatorname{Av}_{R}(\phi)=L, E \subseteq Q,|E|=k\right\}
\end{gathered}
$$

whenever $1 \leq q<p$ which are given implicitly via certain solutions of related ODE's (see [5]). However Theorem 1 (see ([1]) allows us to treat problems of more general nature and the purpose of this paper is to present certain applications of this method in the case of Lorentz type estimates.

Our first application is related to multiple weak-type estimates and is described in the following

Theorem 2. Given the real numbers $f, F_{1}, \ldots, F_{m}>0$ and $p_{1}, \ldots, p_{m}>1$ with $f \leq \min \left\{\left(\frac{p_{j}}{p_{j}-1} F_{j}\right)^{1 / p_{j}}: 1 \leq j \leq m\right\}$ and given any nondecreasing $G:[0,+\infty) \rightarrow$ $[0,+\infty)$ and $h:(0,1] \rightarrow \mathbb{R}^{+}$be any locally integrable function, we have for the following Bellman type function

$$
\begin{aligned}
\mathcal{B}_{G, h, p_{1}, \ldots, p_{m}}^{\mathcal{T}}\left(F_{1}, \ldots F_{m}, f, k\right) & =\sup \left\{\int_{0}^{k} G\left[\left(M_{\mathcal{T}} \phi\right)^{*}(t)\right] h(t) d t: \phi \geq 0 \text { measurable on } X\right. \\
\text { with }\|\phi\|_{1} & \left.=f,\|\phi\|_{p_{1}, \infty} \leq F_{1}, \ldots,\|\phi\|_{p_{m}, \infty} \leq F_{m}\right\}
\end{aligned}
$$

the equality

$$
\mathcal{B}_{G, h, p_{1}, \ldots, p_{k}}^{\mathcal{T}}\left(F_{1}, \ldots F_{k}, f, k\right)=\int_{0}^{k} G\left(\frac{1}{t} \int_{0}^{\min (t, \sigma\}} \min _{1 \leq j \leq m}\left(\frac{F_{j}}{u}\right)^{1 / p_{j}} d u\right) h(t) d t
$$

where $\sigma$ is defined by the equality

$$
\int_{0}^{\sigma} \min _{1 \leq j \leq m}\left(\frac{F_{j}}{u}\right)^{1 / p_{j}} d u=f
$$

Using the above Theorem we find the $L^{p, \infty} \rightarrow L^{q, r}$ Lorentz type Bellman function for the maximal operator. To make the result more readable let us denote by $p^{\prime}, q^{\prime}$ the dual exponents of $p, q>1\left(\right.$ so $\left.p^{\prime}=\frac{p}{p-1}\right)$ 
Theorem 3. Given $1<q<p$ and $r>0$ the Bellman function:

$$
\begin{gathered}
\mathcal{B}_{(p, \infty),(q, r)}^{\mathcal{T}}(F, f, L)=\sup \left\{\left\|\max \left(M_{\mathcal{T}} \phi, L\right)\right\|_{L^{q, r}(X, \mu)}^{r}: \phi \geq 0\right. \text { is measurable with } \\
\left.\|\phi\|_{L^{1}(X, \mu)}=f,\|\phi\|_{L^{p, \infty}(X \mu)}^{p}=F\right\}
\end{gathered}
$$

defined for $0<f<p^{\prime} F^{1 / p}$ and $f \leq L$ is given by

$$
\mathcal{B}_{(p, \infty),(q, r)}^{\mathcal{T}}(F, f, L)=\left\{\begin{array}{l}
\frac{q(p-1) q^{\prime}}{r(p-q)}\left(p^{\prime}\right)^{p^{\prime} r / q^{\prime}} f^{\frac{r(p-q)}{q(p-1)}} F^{\frac{r(q-1)}{q(p-1)}}+\frac{q}{r} L^{r}-\frac{q}{r} q^{\prime} f^{\frac{r}{q}} L^{\frac{r}{q}(q-1)} \\
\text { when } L \leq\left(p^{\prime}\right)^{p^{\prime}}\left(\frac{F}{f}\right)^{1 /(p-1)} \\
\frac{q\left(p^{\prime}\right)^{r p / q}}{r\left(\frac{p}{q}-1\right)} F^{\frac{r}{q}} L^{r\left(1-\frac{p}{q}\right)}+L^{r} \\
\text { when } L \geq\left(p^{\prime}\right)^{p^{\prime}}\left(\frac{F}{f}\right)^{1 /(p-1)}
\end{array}\right.
$$

The proofs of the above two Theorems are given in section 3 .

Next we define the Bellman function related to a Lorentz $L^{p, q} \rightarrow L^{p, q}$ type estimate for the (martingale) maximal operator, where $p, \dot{q}>1$ are arbitrary

$$
\begin{gathered}
\mathcal{B L}_{p, q}^{\mathcal{T}}(F, f)=\sup \left\{\left\|M_{\mathcal{T}} \phi\right\|_{L^{p, q}(X, \mu)}^{q}: \phi \geq 0\right. \text { is measurable with } \\
\left.\|\phi\|_{L^{1}(X, \mu)}=f,\|\phi\|_{L^{p, q}(X \mu)}^{q}=F\right\} .
\end{gathered}
$$

In 11 it has been proved that $M_{\mathcal{T}}$ satisfies an $L^{p, q} \rightarrow L^{p, q}$ estimate with best constant $p^{\prime}$. Here we will determine the exact form of the corresponding Bellman function (1.11). We have.

Theorem 4. The Bellman function (1.11) is defined for all pairs $(F, f)$ with (i) $0<f^{q} \leq\left(\frac{p^{\prime}}{q^{\prime}}\right)^{q-1} F$ if $1<p \leq q$ and (ii) $0<f^{q} \leq \frac{q}{p} F$ if $1<q<p$ and in both cases it is given by

$$
\mathcal{B L}_{p, q}^{\mathcal{T}}(F, f)=\left(\frac{p^{\prime}}{q^{\prime}}\right)^{q} \omega_{q}\left(\left(\frac{q^{\prime}}{p^{\prime}}\right)^{q-1} \frac{f^{q}}{F}\right)^{q} F .
$$

Here $\omega_{q}:[0,1] \rightarrow\left[1, q^{\prime}\right]$ is the inverse function of $H_{q}(z)=-(q-1) z^{q}+q z^{q-1}$ (defined on $\left[1, q^{\prime}\right]$ ) thus the same function as the one appearing in the Bellman functions of the usual $L^{p}$ norms. Note though that in the case $1<q<p$ only a restriction of $H_{q}$ is inverted (see the proof of this theorem). Also we note that the case $q=1$ could be inferred from this Theorem but it is easy to see that since $\int_{0}^{1} t^{\frac{1}{p}-2} \int_{0}^{t} g(u) d u d t=p^{\prime} \int_{0}^{1}\left(t^{\frac{1}{p}-1}-1\right) g(t) d t$ that from Theorem $1 \mathcal{B L}_{p, 1}^{\mathcal{T}}(F, f)=$ $p^{\prime}(F-f)$. In section 4 we will prove Theorem 4 .

\section{TREeS AND MAXIMAL OPERATORS}

As in [4] we let $(X, \mu)$ be a nonatomic probability space (i.e. $\mu(X)=1)$. Two measurable subsets $A, B$ of $X$ will be called almost disjoint if $\mu(A \cap B)=0$. Then we give the following.

Definition 1. A set $\mathcal{T}$ of measurable subsets of $X$ will be called a tree if the following conditions are satisfied:

(i) $X \in \mathcal{T}$ and for every $I \in \mathcal{T}$ we have $\mu(I)>0$.

(ii) For every $I \in \mathcal{T}$ there corresponds a finite subset $\mathcal{C}(I) \subseteq \mathcal{T}$ containing at least two elements such that: 
(a) the elements of $\mathcal{C}(I)$ are pairwise almost disjoint subsets of $I$,

(b) $I=\bigcup \mathcal{C}(I)$.

(iii) $\mathcal{T}=\bigcup_{m \geq 0} \mathcal{T}_{(m)}$ where $\mathcal{T}_{(0)}=\{X\}$ and $\mathcal{T}_{(m+1)}=\bigcup_{I \in \mathcal{T}_{(m)}} \mathcal{C}(I)$.

(iv) We have $\lim _{m \rightarrow \infty} \sup _{I \in \mathcal{T}_{(m)}} \mu(I)=0$.

By removing the measure zero exceptional set $E(\mathcal{T})=\bigcup_{I \in \mathcal{T}} \bigcup_{\substack{J_{1}, J_{2} \in \mathcal{C}(I) \\ J_{1} \neq J_{2}}}\left(J_{1} \cap J_{2}\right)$ we may replace the almost disjointness above by disjointness.

Now given any tree $\mathcal{T}$ we define the maximal operator associated to it as follows

$$
M_{\mathcal{T}} \phi(x)=\sup \left\{\frac{1}{\mu(I)} \int_{I}|\phi| d \mu: x \in I \in \mathcal{T}\right\}
$$

for every $\phi \in L^{1}(X, \mu)$.

The above setting can be used not only for the dyadic maximal operator but also for the maximal operator on martingales, hence many of the results here can be viewed as generalizations and refinements of the classical Doob's inequality.

The following Lemma has been proved in [4] and provides the basis of constructing examples that show sharpness.

Lemma 1. For every $I \in \mathcal{T}$ and every $\alpha$ such that $0<\alpha<1$ there exists a subfamily $\mathcal{F}(I) \subseteq \mathcal{T}$ consisting of pairwise almost disjoint subsets of I such that

$$
\mu\left(\bigcup_{J \in \mathcal{F}(I)} J\right)=\sum_{J \in \mathcal{F}(I)} \mu(J)=(1-\alpha) \mu(I) .
$$

Then we have the following Lemma which give the one side of Theorem 1.

Lemma 2. Given any nonnegative integrable $\phi$ on $X$ we have*

$$
\left(M_{\mathcal{T}} \phi\right)^{*}(t) \leq \frac{1}{t} \int_{0}^{t} \phi^{*}(u) d u
$$

for every $t \in(0,1)$ and therefore whenever $G, h, k$ are as in Theorem 1

$$
\int_{0}^{k} G\left[\left(M_{\mathcal{T}} \phi\right)^{*}(t)\right] h(t) d t \leq \int_{0}^{k} G\left(\frac{1}{t} \int_{0}^{t} \phi^{*}(u) d u\right) h(t) d t .
$$

Proof. Fixing $t \in(0,1)$ let $\alpha=\left(M_{\mathcal{T}} \phi\right)^{*}(t)=\inf \left\{\lambda: \mu\left(\left\{M_{\mathcal{T}} \phi \geq \lambda\right\}\right) \leq t\right\}$. Then given any $\lambda<\alpha$ we have $\mu\left(\left\{M_{\mathcal{T}} \phi \geq \lambda\right\}\right)>t$ and using the decomposition of $\left\{M_{\mathcal{T}} \phi \geq \lambda\right\}$ as a disjoint union of elements $I$ of $\mathcal{T}$ maximal under the condition $\int_{I} \phi d \mu \geq \lambda \mu(I)$, we conclude that there exists measurable $A \subseteq X$ with $\mu(A)>t$ and $\int_{A} \phi d \mu \geq \lambda \mu(A)$. But now since $\phi^{*}$ is decreasing we have

$$
\frac{1}{t} \int_{0}^{t} \phi^{*} \geq \frac{1}{\mu(A)} \int_{0}^{\mu(A)} \phi^{*} \geq \frac{1}{\mu(A)} \int_{A} \phi d \mu \geq \lambda .
$$

This holding for any $\lambda<\alpha$ implies (2.3).

The construction in the next Lemma appears also in [11] and provides the other half of Theorem 1. We include a simpler proof for completeness. 
Lemma 3. For $G, h, k$ and $g$ as in Theorem 1, there exists a sequence of measurable functions $\psi_{N}: X \rightarrow \mathbb{R}^{+}$such that $\psi_{N}^{*}=g$ and

$$
\lim \sup _{N \rightarrow \infty} \int_{0}^{k} G\left[\left(M_{\mathcal{T}} \psi_{N}\right)^{*}(t)\right] h(t) d t \geq \int_{0}^{k} G\left(\frac{1}{t} \int_{0}^{t} g\right) h(t) d t .
$$

Proof. Fixing $\alpha$ with $0<\alpha<1$ and using Lemma 1 , we choose for every $I \in \mathcal{T}$ a family $\mathcal{F}(I) \subseteq \mathcal{T}$ of pairwise almost disjoint subsets of $I$ such that

$$
\sum_{J \in \mathcal{F}(I)} \mu(J)=(1-\alpha) \mu(I)
$$

Then we define $\mathcal{S}=\mathcal{S}_{\alpha}$ to be the smallest subset of $\mathcal{T}$ such that $X \in \mathcal{S}$ and for every $I \in \mathcal{S}, \mathcal{F}(I) \subseteq \mathcal{S}$. Next for every $I \in \mathcal{S}$ we define the set

$$
A_{I}=I \backslash \bigcup_{J \in \mathcal{F}(I)} J
$$

and note that $\mu\left(A_{I}\right)=\alpha \mu(I)$ and $I=\bigcup A_{J}$ for every $I \in \mathcal{S}$. Also since $\mathcal{S}=$ $J \in \mathcal{S}$
$J \subseteq I$

$\bigcup_{m \geq 0} \mathcal{S}_{(m)}$ where $\mathcal{S}_{(0)}=\{X\}$ and $\mathcal{S}_{(m+1)} \stackrel{=}{=} \bigcup_{I \in \mathcal{S}_{(m)}} \mathcal{F}(I)$, we can define $\operatorname{rank}(I)=$ $r(I)$ for $I \in \mathcal{S}$ to be the unique integer $m$ such that $I \in \mathcal{S}_{(m)}$ and remark that $\sum_{\substack{\mathcal{S} \ni \ni \subseteq I \\ r(J)=r(\bar{I})+m}} \mu(J)=(1-\alpha)^{m} \mu(I)$ for every $I \in \mathcal{S}$. For any $m \geq 0$ let

$$
\gamma_{m}=\frac{1}{\alpha(1-\alpha)^{m}} \int_{(1-\alpha)^{m+1}}^{(1-\alpha)^{m}} g(u) d u
$$

and for any $I \in \mathcal{S}_{(m)}$ i.e. $\operatorname{rank}(I)=m$, since $\mu$ is nonatomic we can choose a random variable $R_{I}: A_{I} \rightarrow[0,+\infty)$ on the probability space $\left(A_{I}, \frac{1}{\mu\left(A_{I}\right)} \mu\right)$ having the same distribution as the restriction of $g$ on the probability space $\left((1-a)^{m+1},(1-\alpha)^{m}\right.$ ] with measure $\frac{1}{\alpha(1-\alpha)^{m}} d \lambda$ ( $\lambda$ being Lebesgue measure). Then define

$$
\phi_{\alpha}(x)=R_{I}(x) \text { when } x \in A_{I}, I \in \mathcal{S} .
$$

For and any $s>0$ the disjointness of the $A_{I}$ 's implies that

$$
\begin{aligned}
\mu\left(\left\{\phi_{\alpha}\right.\right. & \geq s\})=\sum_{I \in \mathcal{S}} \mu\left(\left\{x \in A_{I}: R_{I}(x) \geq s\right\}\right)= \\
& =\sum_{m \geq 0} \sum_{I \in \mathcal{S}_{(m)}} \frac{\mu\left(A_{I}\right)}{\alpha(1-\alpha)^{m}}\left|\left\{t \in\left((1-a)^{m+1},(1-\alpha)^{m}\right]: g(t) \geq s\right\}\right|= \\
& =|\{t \in(0,1]: g(t) \geq s\}|
\end{aligned}
$$

hence $\phi_{\alpha}$ and $g$ have the same distribution and since $g$ is nonincreasing and right continuous on $(0, \mu(X)]$ we conclude that $\phi_{\alpha}^{*}=g$. Moreover

$$
\frac{1}{\mu\left(A_{I}\right)} \int_{A_{I}} \phi_{a} d \mu=\gamma_{m}
$$


for every $I \in \mathcal{S}$ with $\operatorname{rank}(I)=m$ and thus

$$
\begin{aligned}
\operatorname{Av}_{I}\left(\phi_{\alpha}\right) & =\frac{1}{\mu(I)} \int_{I} \phi_{a} d \mu=\frac{1}{\mu(I)} \sum_{\substack{J \in \mathcal{S} \\
J \subseteq I}} \int_{A_{J}} \phi_{a} d \mu= \\
& =\frac{\alpha}{\mu(I)} \sum_{\ell \geq 0} \gamma_{\ell+\operatorname{rank}(I)} \sum_{\substack{\mathcal{S} \ni J \subseteq I \\
\operatorname{rank}(J)=\operatorname{rank}(I)+\ell}} \mu(J)= \\
& =\alpha \sum_{\ell \geq 0} \gamma_{\ell+m}(1-\alpha)^{\ell}=\frac{1}{(1-\alpha)^{m}} \int_{0}^{(1-\alpha)^{m}} g(u) d u
\end{aligned}
$$

implying that $M_{\mathcal{T}} \phi \geq \frac{1}{(1-\alpha)^{m}} \int_{0}^{(1-\alpha)^{m}} g(u) d u$ on the set $\bigcup_{I \in \mathcal{S}: r(I)=m} I$ which has measure $(1-\alpha)^{m}$ and thus $\left(M_{\mathcal{T}} \phi_{\alpha}\right)^{*}(t) \geq \frac{1}{(1-\alpha)^{m}} \int_{0}^{(1-\alpha)^{m}} g(u) d u$ for every $t \in$ $\left((1-a)^{m+1},(1-\alpha)^{m}\right)$.

Now with $N$ large, taking $\alpha_{N}=1-(1-k)^{1 / N}$ and $\psi_{N}=\phi_{\alpha_{N}}$ we have

$\int_{0}^{k} G\left[\left(M_{\mathcal{T}} \psi_{N}\right)^{*}(t)\right] h(t) d t \geq \sum_{j \geq 0} G\left(\frac{1}{(1-k)^{1+\frac{m}{N}}} \int_{0}^{(1-k)^{1+\frac{m}{N}}} g(u) d u\right) \int_{(1-k)^{1+\frac{m+1}{N}}}^{(1-k)^{1+\frac{m}{N}}} h(t) d t$

the last sum converging to $\int_{0}^{k} G\left(\frac{1}{t} \int_{0}^{t} g\right) h(t) d t$ as $N \rightarrow \infty$ by monotone convergence. This completes the proof.

\section{THE CASE OF WEAK TYPE CONDITIONS}

Here we will prove Theorems 2 and 3. Theorem 2 follows from the following more general Proposition by taking $R(t)$ to be the decreasing function $\min _{1 \leq j \leq m}\left(\frac{F_{j}}{t}\right)^{1 / p_{j}}$.

Proposition 1. Let $R:(0,1] \rightarrow(0,+\infty)$ be a decreasing, continuous and integrable function and for any $f$ with $0<f \leq \int_{0}^{1} R(t) d t$ let $\sigma=\sigma(f)$ be the unique number in $(0,1]$ with $\int_{0}^{\sigma} R(t) d t=f$. Then for any $(X, \mu, \mathcal{T}), G, h, k$ as in Theorem 1 we have

$$
\begin{gathered}
\sup \left\{\int_{0}^{k} G\left[\left(M_{\mathcal{T}} \phi\right)^{*}(t)\right] h(t) d t: \phi \geq 0 \text { with } \int_{X} \phi d \mu=f \text { and } \phi^{*} \leq R\right\}= \\
=\int_{0}^{k} G\left(\frac{1}{t} \int_{0}^{t} R(u) \chi_{[0, \sigma(f))}(u) d u\right) h(t) d t .
\end{gathered}
$$

Proof. By Theorem the above type supremum but fixing $\phi^{*}=g$ is equal to $\int_{0}^{k} G\left(\frac{1}{t} \int_{0}^{t} g(u) d u\right) h(t) d t$.

But we must have $g(t) \leq R(t)$ for any $t$ and $\int_{0}^{1} g=\int_{X} \phi d \mu=f=\int_{0}^{\sigma} R$ we conclude that one the one hand $\int_{0}^{t} g \leq \int_{0}^{t} R$ when $0 \leq t \leq \sigma$ and on the other hand $\int_{0}^{t} g \leq \int_{0}^{1} g=\int_{0}^{\sigma} R=\int_{0}^{t} R(u) \chi_{[0, \sigma(f))}(u) d u$ when $\sigma<t \leq 1$ we get $G\left(\frac{1}{t} \int_{0}^{t} g\right) \leq G\left(\frac{1}{t} \int_{0}^{t} R \chi_{[0, \sigma)}\right)$ for all $t$. Thus using the converse implication in Theorem 1 for the decreasing right continuous function $R \chi_{[0, \sigma)}$ completes the proof of (3.1). 
Now to prove Theorem 3 we remark that using Theorem 2 with $G(x)=\max (x, L)^{r}$, $h(t)=t^{\frac{r}{q}-1}$ and $m=1, p_{1}=p$ that the expression $\mathcal{B}_{(p, \infty),(q, r)}^{\mathcal{T}}(F, f, L)$ in (1.9) is equal to the following expression (actually we get the supremum under $\|\phi\|_{L^{p, \infty}(X \mu)}^{p} \leq$ $F$ but it is easy to see that at the extremum above we have the equality $\|\phi\|_{L^{p, \infty}(X \mu)}^{p}=$ F)

$$
\int_{0}^{1} t^{\frac{r}{q}-1} \max \left(\frac{1}{t} \int_{0}^{\min (t, \sigma\}}\left(\frac{F}{u}\right)^{1 / p} d u, L\right)^{r} d t
$$

where $\sigma=\sigma_{p}(F, f)$ is given by $\int_{0}^{\sigma}\left(\frac{F}{u}\right)^{1 / p} d u=f$ thus

$$
\sigma=\left(\frac{f}{p^{\prime} F^{1 / p}}\right)^{p^{\prime}}
$$

Next note that using (3.3)

$$
\frac{1}{t} \int_{0}^{\min (t, \sigma\}}\left(\frac{F}{u}\right)^{1 / p} d u=p^{\prime} F^{1 / p} \frac{1}{t} \min (t, \sigma)^{1-\frac{1}{p}}=\min \left(p^{\prime}\left(\frac{F}{t}\right)^{1 / p}, \frac{f}{t}\right)
$$

and so we have to compute the integral $\int_{0}^{1} t^{\frac{r}{q}-1} \Sigma(t)^{r} d t$ where $\Sigma(t)$ is given by

$$
\Sigma(t)=\max \left(\min \left(p^{\prime}\left(\frac{F}{t}\right)^{1 / p}, \frac{f}{t}\right), L\right)
$$

Observing that $L>\min \left(p^{\prime}\left(\frac{F}{t}\right)^{1 / p}, \frac{f}{t}\right)$ if and only if $t>\frac{f}{L}$ or $t>\left(\frac{p^{\prime}}{L}\right)^{p} F$ we consider two cases:

Case 1 If $\frac{f}{L} \leq\left(\frac{p^{\prime}}{L}\right)^{p} F$ that is $L \leq\left(p^{\prime}\right)^{p^{\prime}}\left(\frac{F}{f}\right)^{\frac{1}{p-1}}$ we easily get $\frac{f}{L} \geq\left(\frac{f}{p^{\prime} F^{1 / p}}\right)^{p^{\prime}}=$ $\sigma$ and therefore we have

$$
\Sigma(t)=\left\{\begin{array}{l}
p^{\prime}\left(\frac{F}{t}\right)^{1 / p} \text { when } 0 \leq t \leq \sigma \\
\frac{f}{t} \text { when } \sigma<t \leq \frac{f}{L} \\
L \text { when } \frac{f}{L}<t \leq 1
\end{array}\right.
$$

and then computing the corresponding integral $\int_{0}^{1} t^{\frac{r}{q}-1} \Sigma(t)^{r} d t$ we get the upper half in (1.11).

Case 2 If $\frac{f}{L}>\left(\frac{p^{\prime}}{L}\right)^{p} F$ that is $L>\left(p^{\prime}\right)^{p^{\prime}}\left(\frac{F}{f}\right)^{\frac{1}{p-1}}$ we easily get $\left(\frac{p^{\prime}}{L}\right)^{p} F<$ $\left(\frac{f}{p^{\prime} F^{1 / p}}\right)^{p^{\prime}}=\sigma$ and therefore we have

$$
\Sigma(t)=\left\{\begin{array}{l}
p^{\prime}\left(\frac{F}{t}\right)^{1 / p} \text { when } 0 \leq t \leq\left(\frac{p^{\prime}}{L}\right)^{p} F \\
L \text { when }\left(\frac{p^{\prime}}{L}\right)^{p} F<t \leq 1
\end{array}\right.
$$

and then computing the corresponding integral $\int_{0}^{1} t^{\frac{r}{q}-1} \Sigma(t)^{r} d t$ we get the lower half in (1.11).

These cases complete the proof of Theorem 3 . 


\section{Proof of Theorem 4}

In view of Theorem 1 and by setting $\phi^{*}=g$ it suffices to determine the supremum of the expression $\Delta(g)=\int_{0}^{1}\left(t^{\frac{1}{p}-1} \int_{0}^{t} g(u) d u\right)^{q} \frac{d t}{t}$ when $g$ runs over all nonnegative decreasing right continuous functions on $(0,1]$ satisfying $\int_{0}^{1} g(t) d t=f$ and $\int_{0}^{1}\left(t^{\frac{1}{p}} g(t)\right)^{q} \frac{d t}{t}=F$. Considering first any bounded such function $g$ we compute by integration by parts

$$
\begin{aligned}
& \int_{0}^{1} t^{q\left(\frac{1}{p}-1\right)}\left(\int_{0}^{t} g(u) d u\right)^{q-1} g(t) d t=\frac{1}{q} \int_{0}^{1} t^{q\left(\frac{1}{p}-1\right)}\left[\left(\int_{0}^{t} g(u) d u\right)^{q}\right]^{\prime} d t= \\
= & \frac{1}{q}\left(\int_{0}^{1} g(u) d u\right)^{q}+\left(1-\frac{1}{p}\right) \int_{0}^{1}\left(t^{\frac{1}{p}-1} \int_{0}^{t} g(u) d u\right)^{q} \frac{d t}{t}=\frac{f^{q}}{q}+\frac{1}{p^{\prime}} \Delta(g) .
\end{aligned}
$$

But using Young's inequality $x y \leq \frac{x^{q}}{q}+\frac{y^{q^{\prime}}}{q^{\prime}}$ in the first integral as follows, where $\gamma=\frac{1}{p}-\frac{1}{q}$ and $\lambda>0$ will be determined later

$$
\begin{gathered}
\int_{0}^{1} t^{q\left(\frac{1}{p}-1\right)}\left(\int_{0}^{t} g(u) d u\right)^{q-1} g(t) d t=\int_{0}^{1} t^{-\gamma+q\left(\frac{1}{p}-1\right)}\left(\frac{1}{\lambda^{1 /(q-1)}} \int_{0}^{t} g(u) d u\right)^{q-1}\left(\lambda g(t) t^{\gamma}\right) d t \leq \\
\quad \leq \frac{1}{q} \int_{0}^{1} \lambda^{q} g(t)^{q} t^{\gamma q} d t+\frac{1}{q^{\prime}} \int_{0}^{1} \lambda^{-q^{\prime}} t^{\left[-\gamma+q\left(\frac{1}{p}-1\right)\right] q^{\prime}}\left(\int_{0}^{t} g(u) d u\right)^{q} d t= \\
=\frac{\lambda^{q}}{q} \int_{0}^{1} g(t)^{q} t^{\frac{q}{p}-1} d t+\frac{\lambda^{-q^{\prime}}}{q^{\prime}} \int_{0}^{1} t^{\frac{q}{p}-q-1}\left(\int_{0}^{t} g(u) d u\right)^{q} d t=\frac{\lambda^{q}}{q} F+\frac{\lambda^{-q^{\prime}}}{q^{\prime}} \Delta(g) .
\end{gathered}
$$

Therefore we have by writing $\delta=\frac{p^{\prime}}{q^{\prime}}$ and taking $\lambda^{q^{\prime}}=(\beta+1) \delta, \beta>0$ and using the above inequalities that

$$
\frac{\beta}{p^{\prime}(\beta+1)} \Delta(g)=\left(\frac{1}{p^{\prime}}-\frac{\lambda^{-q^{\prime}}}{q^{\prime}}\right) \Delta(g) \leq \frac{\lambda^{q} F-f}{q}
$$

and so

$$
\Delta(g) \leq \delta \frac{\beta+1}{\beta} \frac{(\beta+1)^{q-1} \delta^{q-1} F-f^{q}}{q-1} .
$$

Next, given an arbitrary $g$, the above estimate can be used for the truncations $g_{M}=\min (g, M)$ and $F, f$ replaced by the corresponding quantities for $g_{M}$ and then take $M \rightarrow+\infty$ and use monotone convergence to infer that (4.1) holds for the general nonnegative decreasing right continuous function on $(0,1]$ satisfying $\int_{0}^{1} g(t) d t=f$ and $\int_{0}^{1}\left(t^{\frac{1}{p}} g(t)\right)^{q} \frac{d t}{t}=F$.

As has been also remarked in 4 it is easy to see that the right hand side of (4.1) is minimized when $\beta$ satisfies the equation $H_{q}(\beta+1)=\frac{f^{q}}{\delta^{q-1} F} \leq 1$ (which is well known that it is $\leq 1$, but also follows from (4.1) by taking $\beta \rightarrow 0^{+}$) and then for this value of $\beta$ the right hand side of (4.1) becomes $\delta^{q} \omega_{q}\left(\frac{f^{q}}{\delta^{q-1} F}\right)^{q} F$. This proves the inequality $\mathcal{B L}_{p, q}^{\mathcal{T}}(F, f) \leq \delta^{q} \omega_{q}\left(\frac{f^{q}}{\delta^{q-1} F}\right)^{q} F$.

Now we consider the continuous positive decreasing function

$$
g_{\alpha}(t)=f(1-\alpha) t^{-\alpha}
$$


where $0 \leq \alpha<1$. Clearly $\int_{0}^{1} g_{\alpha}(t) d t=f$ and since $\frac{1}{t} \int_{0}^{t} g_{\alpha}(u) d u=\frac{g_{\alpha}(t)}{1-\alpha}$ for all $t \in$ $(0,1]$ we have $\Delta\left(g_{\alpha}\right)=\left(\frac{1}{1-\alpha}\right)^{q} \int_{0}^{1}\left(t^{\frac{1}{p}-1} g_{\alpha}(t)\right)^{q} \frac{d t}{t}$. The condition $\int_{0}^{1}\left(t^{\frac{1}{p}} g_{\alpha}(t)\right)^{q} \frac{d t}{t}=$ $F$ is then equivalent to the equation in $\alpha$

$$
\frac{p}{q} \frac{(1-\alpha)^{q}}{1-\alpha p}=\frac{F}{f^{q}} .
$$

Consider the function $w(\alpha)=\frac{p}{q} \frac{(1-\alpha)^{q}}{1-\alpha p}$ defined on $0 \leq \alpha<\frac{1}{p}$. We have $w^{\prime}(\alpha)=$ $\frac{p(q-1) \alpha+p-q}{1-\alpha} w(\alpha), w(0)=\frac{p}{q}$ and $\lim _{\alpha \rightarrow(1 / p)^{+}} w(\alpha)=+\infty$. Now consider the following cases:

Case $11<p \leq q$.In this case the function $w$ has a minimum at $\alpha_{0}=\frac{q-p}{p(q-1)}<\frac{1}{p}$ and is strictly increasing on $\left[\alpha_{0}, \frac{1}{p}\right)$ and since also $\alpha_{0}=1-\frac{q^{\prime}}{p^{\prime}}=1-\frac{1}{\delta}$ which gives $w\left(\alpha_{0}\right)=\delta^{q-1}$, its range is $\left[\delta^{q-1},+\infty\right)$. This implies that the domain of $\mathcal{B L}_{p, q}^{\mathcal{T}}(F, f)$ in this case consists of all $F, f$ with $0<f^{q} \leq \delta^{q-1} F$ as asserted in Theorem 4, and with such a pair $(F, f)$ there exists a unique $\alpha=\alpha(F, f)$ in the interval $\left[\alpha_{0}, \frac{1}{p}\right)$ such that $w(a)=\frac{F}{f^{q}}$. Then for this $\alpha$ we have $\int_{0}^{1} g_{\alpha}(t) d t=f, \int_{0}^{1}\left(t^{\frac{1}{p}} g_{\alpha}(t)\right)^{q} \frac{d t}{t}=F$ and $\Delta\left(g_{\alpha}\right)=\left(\frac{1}{1-\alpha}\right)^{q} F$ and since $\frac{1}{1-\alpha} \geq \frac{1}{1-\alpha_{0}}=\delta$ we may write $\frac{1}{1-\alpha}=\delta z$ with $z \geq 1$ (and also $z<\frac{1}{\delta} p^{\prime}=q^{\prime}$ ) and then it is easy to see that (4.3) transforms into $H_{q}(z)=\frac{f^{q}}{\delta^{q-1} F}$ and so $z=\omega_{q}\left(\frac{f^{q}}{\delta^{q-1} F}\right)$ giving that $\Delta\left(g_{\alpha}\right)=\delta^{q} \omega_{q}\left(\frac{f^{q}}{\delta^{q-1} F}\right)^{q} F$ and thus proving Theorem 4 in this case.

Case $21<q<\dot{p}$.In this case the function $w$ has positive derivative hence it is one to one and its range is $\left[\frac{p}{q},+\infty\right)$. On the other hand for any nonnegative decreasing right continuous function $g$ on $(0,1]$ satisfying $\int_{0}^{1} g(t) d t=f$ and $\int_{0}^{1}\left(t^{\frac{1}{p}} g(t)\right)^{q} \frac{d t}{t}=F$, Chebyshev's inequality (applicable since $t^{\frac{q}{p}-1}$ is here decreasing) gives

$$
F=\int_{0}^{1} g(t)^{q} t^{\frac{q}{p}-1} d t \geq \int_{0}^{1} g(t)^{q} d t \int_{0}^{1} t^{\frac{q}{p}-1} d t \geq\left(\int_{0}^{1} g(t) d t\right)^{q} \int_{0}^{1} t^{\frac{q}{p}-1} d t=f^{q} \frac{p}{q}
$$

therefore on the one hand this proves that the domain of $\mathcal{B L}_{p, q}^{\mathcal{T}}(F, f)$ in this case consists of all $F, f$ with $0<f^{q} \leq \frac{q}{p} F$ as asserted in Theorem 4 , and on the other hand given any pair $(F, f)$ satisfying $\frac{F}{f^{q}} \geq \frac{p}{q}$ in (4.3) there exists a unique $\alpha=$ $\alpha(F, f)$ in the interval $\left[0, \frac{1}{p}\right)$ such that $w(a)=\frac{F}{f^{q}}$. Since in this case $\delta=\frac{p^{\prime}}{q^{\prime}}<1$ we may write $\frac{1}{1-\alpha}=\delta z$ with $z \geq \frac{1}{\delta}>1$ (and also $z<\frac{1}{\delta} p^{\prime}=q^{\prime}$ ) and then as in case 1 we get for this $\alpha, \int_{0}^{1} g_{\alpha}(t) d t=f, \int_{0}^{1}\left(t^{\frac{1}{p}} g_{\alpha}(t)\right)^{q} \frac{d t}{t}=F$ and $\Delta\left(g_{\alpha}\right)=\delta^{q} \omega_{q}\left(\frac{f^{q}}{\delta^{q-1} F}\right)^{q} F$. The only difference here is that only the restriction of $H_{q}$ on $\left[\frac{q^{\prime}}{p^{\prime}}, q^{\prime}\right]$ is inverted. These complete the proof of Theorem 4 .

\section{REFERENCES}

[1] D. L. Burkholder, Martingales and Fourier analysis in Banach spaces, C.I.M.E. Lectures (Varenna (Como), Italy, 1985), Lecture Notes in Mathematics 1206 (1986), 61-108.

[2] D. L. Burkholder, Boundary value problems and sharp inequalities for martingale transforms, Ann. of Prob. 12 (1984), 647-702.

[3] L. Grafakos, S. Montgomery-Smith. Best constants for uncentered maximal functions, Bull. London Math. Soc. 29 (1997), no.1, 60-64. 
[4] A. D. Melas, The Bellman functions of dyadic-like maximal operators and related inequalities, Adv. in Math. 192 (2005), 310-340.

[5] A. D. Melas, Sharp general local estimates for dyadic -like maximal operators and related Bellman functions, Adv. in Math. 220 (2009), 1631-1654.

[6] A. D. Melas, Dyadic-like maximal operators on $L \log L$ functions, Journal of Funct. Anal. 257 no. 6 (2009), 367-426.

[7] A. D. Melas, E. Nikolidakis, A sharp integral rearrangement inequality for the dyadic maximal operator and applications, submitted.

[8] F. Nazarov, S. Treil, The hunt for a Bellman function: applications to estimates for singular integral operators and to other classical problems of harmonic analysis, Algebra i Analyz 8 no. 5 (1996), 32-162.

[9] F. Nazarov, S. Treil, A. Volberg, The Bellman functions and two-weight inequalities for Haar multipliers. Journ. Amer. Math. Soc. 12 no. 4 (1999), 909-928.

[10] F. Nazarov, S. Treil, A. Volberg, Bellman function in Stochastic Optimal Control and Harmonic Analysis (how our Bellman function got its name), Oper. Theory: Advances and Appl. 129 (2001), 393-424, Birkhäuser Verlag.

[11] E. Nikolidakis, The geometry of the maximal operator, Rev. Math.Iber., to appear.

[12] L. Slavin, V. Vasyunin, Sharp results in the integral-form John-Nirenberg inequality, Trans. Amer. Math. Soc. 363, no 8, (2011), 4135-4169.

[13] L. Slavin, A. Volberg. The explicit BF for a dyadic Chang-Wilson-Wolff theorem. The sfunction and the exponential integral. Contmp. Math., 444 (2007), 215-228.

[14] E. M. Stein. Harmonic Analysis, Princeton Mathematical Series 43, (1993), Princeton University Press.

[15] L. Slavin, A. Stokolos, V. Vasyunin. Monge-Ampère equations and Bellman functions: The dyadic maximal operator. C. R. Acad. Paris Sér. I Math., 346, no 9-10, (2008), 585-588.

[16] V. Vasyunin. The sharp constant in the reverse Holder inequality for Muckenhoupt weights. Algebra i Analiz, 15 (2003), no. 1, 73-117

[17] V. Vasyunin, A. Volberg. The Bellman functions for a certain two weight inequality: the case study. Algebra i Analiz, 18 (2006), No. 2

[18] V. Vasyunin, A. Volberg. Monge-Ampere equation and Bellman optimization of Carleson embedding Theorem, Amer. Math. Soc. Translations Series 2, 226, (2009).

[19] A. Volberg, Bellman approach to some problems in Harmonic Analysis, Seminaire des Equations aux derivées partielles, Ecole Polytéchnique, 2002, eposé. XX, 1-14.

[20] G. Wang, Sharp maximal inequalities for conditionally symmetric martingales and Brownian motion, Proc. Amer. Math. Soc. 112 (1991), 579-586.

Department of Mathematics, University of Athens, Panepistimiopolis 15784, Athens, Greece

E-mail address: amelas@math.uoa.gr, lefteris@math.uoc.gr 